\title{
REGULARITY AND UNIQUENESS OF P-HARMONIC MAPS WITH SMALL RANGE
}

\author{
ALI FARDOUN AND RACHID REGBAOUI
}

\begin{abstract}
We prove the uniqueness of solutions to Dirichlet problem for $p$ harmonic maps with images in a small geodesic ball of the target manifold. As a consequence, we show that such maps have Hölder continuous derivatives. This gives an extension of a result by $\mathrm{S}$. Hildebrandt et al [7] concerning harmonic maps.
\end{abstract}

\section{INTRODUCTION}

Let $(M, g)$ and $(N, h)$ be compact Riemannian manifolds with dimensions $m \geq 2$ and $n \geq 2$ respectively. If $p \geq 2$, the $p$-energy of a map $u \in C^{1}(M, N)$ is defined by

$$
E_{p}(u)=\frac{1}{p} \int_{M}|d u|^{p} d g
$$

where $d g$ denotes the volume element of the metric $g$, and for each $x \in M, d u(x)$ : $T_{x} M \rightarrow T_{u(x)} N$ is the differential of $u$ at $x$. Here the norm $|d u(x)|$ of the differential of $u$ is given by

$$
|d u(x)|^{2}=g^{\alpha \beta}(x) h_{i j}(u(x)) \frac{\partial u^{i}}{\partial x_{\alpha}}(x) \frac{\partial u^{j}}{\partial x_{\beta}}(x),
$$

where $\left(g^{\alpha \beta}\right)$ is the inverse matrix of the metric $g$ in local coordinates $\left(x_{1}, \cdots, x_{m}\right)$ on $M$, and in local coordinates on $\mathrm{N},\left(u^{1}, \cdots, u^{n}\right)$ are the components of $u$, and $\left(h_{i j}\right)$ is the matrix of the metric $h$.

$P$-harmonic maps are critical points of the functional $E_{p}$. If $u$ is a smooth $p$ harmonic map, say $C^{2}$, then it satisfies the following system of partial differential equations

$$
-\operatorname{div}\left(|d u|^{p-2} \nabla u^{j}\right)=|d u|^{p-2} \Gamma_{k l}^{j}(u) \nabla u^{k} \nabla u^{l}, j=1, \cdots, n,
$$

where $\Gamma_{k l}^{j}$ are the Christoffel symbols of the metric $h$. Here div and $\nabla$ denote respectively the divergence and the gradient with respect to the metric $g$ on $M$. In particular, the notation $\nabla u^{k} \nabla u^{l}$ stands for the Riemannian inner product of $\nabla u^{k}$ and $\nabla u^{l}$ with respect to $g$, that's,

$$
\nabla u^{k} \nabla u^{l}=g^{\alpha \beta} \frac{\partial u^{k}}{\partial x_{\alpha}} \frac{\partial u^{l}}{\partial x_{\beta}} .
$$


The variational approach to the existence of $p$-harmonic maps imposes a larger functions space than $C^{1}(M, N)$ for the $p$-energy functional. In order to give a more precise definition of critical points of the functional $E_{p}$, let us recall some definitions. Without loss of generality, we may suppose that $(N, h)$ is isometrically embedded in some Euclidean space $\mathbb{R}^{k}$. Then for any $p \geq 2$, we define the Sobolev space

$$
W^{1, p}(M, N):=\left\{u \in W^{1, p}\left(M, \mathbb{R}^{k}\right): u(x) \in N \text { a.e }\right\} .
$$

The $p$-energy can be extended to maps $u=\left(u^{1}, \cdots, u^{k}\right) \in W^{1, p}(M, N)$ by

$$
E_{p}(u)=\frac{1}{p} \int_{M}|\nabla u|^{p} d g
$$

where $|\nabla u|^{p}=\left(\sum_{j=1}^{k}\left|\nabla u^{j}\right|^{2}\right)^{\frac{p}{2}}$, and $\nabla u^{j}$ is the gradient of $u^{j}$ with respect to the metric $g$ (and its norm $\left|\nabla u^{j}\right|$ is also taken with respect to $g$ ).

$P$-harmonic maps are critical points $u \in W^{1, p}(M, N)$ of the functional $E_{p}$ with respect to variations of the form

$$
\Pi_{N}(u+t \varphi),
$$

where $t \in(-\varepsilon, \varepsilon)$ with $\varepsilon>0, \varphi \in C_{0}^{\infty}\left(M, \mathbb{R}^{k}\right)$ and $\Pi_{N}$ is the nearest point projection from a tubular neigborhood of $N$ in $\mathbb{R}^{k}$ onto $N$. Then we get the Euler-Lagrange system (in the distributional sense):

$$
-\operatorname{div}\left(|\nabla u|^{p-2} \nabla u\right)=|\nabla u|^{p-2} A(u)(\nabla u, \nabla u),
$$

where $A(u)$ is the second fundamental form of $N$ at $u$, and we are using the notation :

$$
A(u)(\nabla u, \nabla u):=g^{\alpha \beta} A(u)\left(\frac{\partial u}{\partial x_{\alpha}}, \frac{\partial u}{\partial x_{\beta}}\right) .
$$

If in addition one allows variations of the form $u \circ \varphi_{t}$, where $\left(\varphi_{t}\right)_{t \in(-\varepsilon, \varepsilon)}$ is a family of smooth transformations of $M$ such that $\varphi_{0}=I_{M}$, one gets the so-called stationary $p$-harmonic maps. A map $u \in W^{1, p}(M, N)$ is a minimizing $p$-harmonic map on a domain $\Omega \subset M$ if, for any $w \in W^{1, p}(\Omega, N)$ agreeing with $u$ on $\partial \Omega$, we have

$$
\int_{\Omega}|\nabla u|^{p} d g \leq \int_{\Omega}|\nabla w|^{p} d g
$$

It is not difficult to see that minimizing maps are stationary $p$-harmonic maps.

The difference between system of equations (1.1) and system (1.2) is that in (1.1) one needs that the image of the solution $u$ lie (locally) in a single system of coordinates on the target manifold $N$, which is not necessary concerning system $(1.2)$. However, one can easily see that for $p$-harmonic maps whose images lie in a 
single local chart of $N$, systems (1.1) and (1.2) are equivalent. In particular, this is the case for continuous $p$-harmonic maps.

Solutions of (1.2) have to be understood in the weak sense, that's, a map $u \in$ $W^{1, p}(M, N)$ is a solution of $(1.2)$ if for any $\varphi \in W_{0}^{1, p}\left(M, \mathbb{R}^{k}\right) \cap L^{\infty}\left(M, \mathbb{R}^{k}\right)$, we have

$$
\int_{M}|\nabla u|^{p-2} \nabla u \cdot \nabla \varphi d g=\int_{M}|\nabla u|^{p-2} A(u)(\nabla u, \nabla u) \cdot \varphi d g
$$

where the dot denotes the usual inner product in $\mathbb{R}^{k}$ and the notation $\nabla u \cdot \nabla \varphi$ means

$$
\nabla u \cdot \nabla \varphi=g^{\alpha \beta} \frac{\partial u}{\partial x_{\alpha}} \cdot \frac{\partial \varphi}{\partial x_{\beta}} .
$$

System (1.2) presents two principal difficulties. The first one is that it is quasilinear and degenerate (due to the presence of $|\nabla u|^{p-2}$ in the left hand side). The second difficulty comes from the nonlinear term $|\nabla u|^{p-2} A(u)(\nabla u, \nabla u)$ caused by the geometry of $N$. One has to distinguish the case $p=2$, which corresponds to harmonic maps, from the other ones. In fact, when $p=2$ we have an elliptic semilinear system, and the theory of linear elliptic equations applies if one has a good control of the right hand side. The case $p \neq 2$ is more complicated, and one has to take care of the vanishing of the term $\nabla u$.

The regularity of harmonic maps has been a very attractive subject of research the last thirty years. The first result is due to S. Hildebrandt et al [7] who proved that harmonic maps $(p=2)$ whose images lie in a small geodesic ball of the target manifold are smooth. Later, W. Jäger and H. Kaul [9] proved the uniqueness of solutions to the Dirichlet problem for such maps. As it can be seen by counterexamples of sphere-valued maps, the Dirichlet problem for general harmonic maps may admit more than one solution. Concerning partial regularity for harmonic maps, many results were obtained for minimizing harmonic maps, and more generally for stationary harmonic maps. Such maps are smooth outside a closed singular set of Hausdorff dimension at most $m-2$, see [1] , 6], [11, [12] and references therein. Concerning $p$-harmonic maps, the situation is more complicated since the system of equations (1.2) is quasilinear and degenerate. To our knowledge, the only known uniqueness result for Dirichlet problems of $p$-harmonic maps is due to the first author [2], and it concerns maps with values in the Euclidean sphere $\mathbb{S}^{n}$. Concerning the partial regularity, it was shown by R. Hardt and F. Lin [5], and S. Luckhauss [10], that minimizing $p$-harmonic maps are $C^{1, \alpha}, 0<\alpha<1$, outside a closed singular set of Hausdorff dimension at most $m-[p]-1$. In [3], M. Fuchs generalised the result of S. Hildebrandt et al [7] above to $p$-harmonic maps but with the additional assumption that the map is stationary. (see also [4, [13] for related results on removable singularities of $p$-harmonic maps).

In this paper we prove the uniqueness of solutions to the Dirichlet problem for $p$-harmonic maps with values in small geodesic balls of the target manifold. As 
a consequence, we prove that such maps are $C^{1, \alpha}$ for some $0<\alpha<1$ and are minimizing the $p$-energy.

Theorem 1.1. Let $M$ be a compact Riemannian manifold with smooth boundary $\partial M, N$ a compact Riemannian manifold and $p \geq 2$. There is a constant $\varepsilon_{0}=$ $\varepsilon_{0}(N, p)>0$ depending only on $N$ and $p$ such that if $u, v \in W^{1, p}(M, N)$ are $p$ harmonic maps satisfying

$$
u(M) \subset B\left(P_{0}, \varepsilon_{0}\right), v(M) \subset B\left(P_{0}, \varepsilon_{0}\right) \text { and } u=v \text { on } \partial M,
$$

where $B\left(P_{0}, \varepsilon_{0}\right)$ is a geodesic ball of $N$ of radius $\varepsilon_{0}$ centered at some point $P_{0} \in N$, then $u=v$ on $M$.

Theorem 1.1 allows us to prove the following regularity result :

Theorem 1.2. Let $M$ and $N$ be compact Riemannian manifolds, and let $p \geq 2$. There is a constant $\varepsilon_{1}=\varepsilon_{1}(N, p)>0$ depending only on $N$ and $p$ such that if $u \in W^{1, p}(M, N)$ is a p-harmonic map satisfying

$$
u(\Omega) \subset B\left(P_{0}, \varepsilon_{1}\right),
$$

where $\Omega$ is an open set of $M$ and $B\left(P_{0}, \varepsilon_{1}\right)$ is a geodesic ball of $N$ of radius $\varepsilon_{1}$ centered at some point $P_{0} \in N$, then $u \in C^{1, \alpha}(\Omega, N)$ for some $0<\alpha<1$. Moreover, $u$ is minimizing the p-energy in $\Omega$ among maps agreeing with $u$ on $\partial \Omega$ and having their values in $B\left(P_{0}, \varepsilon_{1}\right)$.

Theorem 1.1 and Theorem 1.2 can be seen respectively as extensions of the results of W. Jäger and $\mathrm{H}$. Kaul [9] and S. Hildebrandt et al [7] above to $p$ harmonic maps. Due to the lack of ellipticity, the regularity $C^{1, \alpha}$ in Theorem 1.2 is the best one could expect in general for $p$-harmonic maps.

It would be interesting to compute the optimal value of the constant $\varepsilon_{0}$ in Theorem [1.1, and $\varepsilon_{1}$ in Theorem [1.2. When $p=2$, S. Hildebrandt et al [7] and W. Jäger and H. Kaul [9] computed respectively upper bounds of the constant $\varepsilon_{0}$ and $\varepsilon_{1}$, they found

$$
\varepsilon_{0}=\varepsilon_{1}<\inf \left(i_{N}, \frac{\pi}{2 \kappa}\right)
$$

where $\kappa>0$ is an upper bound of the sectional curvature of $N$, and $i_{N}$ is the injectivity radius of $N$. These bounds are optimal as it can be seen by considering sphere-valued harmonic maps (see [8]). For stationary $p$-harmonic maps, M.Fuchs [3] obtained $\varepsilon_{1}<\inf \left(i_{N}, \frac{\pi}{4 \kappa}\right)$. In the case that $N=\mathbb{S}^{n}$, the first author found in [2] the optimal bounds $\varepsilon_{0}=\varepsilon_{1}<\frac{\pi}{2}$.

The paper is organized as follows. In section 2 we prove Theorem 1.1. In section 3 we show the existence of a minimizing $p$-harmonic map with small range, and then we combine this result with Theorem 1.1 to prove Theorem 1.2 . 


\section{UNIQUENESS OF $p$-HARMONIC MAPS WITH SMALL RANGE}

This section is devoted to the proof of Theorem 1.1, which needs some preliminary results. In what follows, $M$ is a compact Riemannian manifold eventually with boundary, and $N$ is a compact Riemannian manifold without boundary, which is isometrically embedded in some Euclidean space $\mathbb{R}^{k}$. For $P_{0} \in N$ and $r>0$, we denote by $B\left(P_{0}, r\right)$ the Euclidean open ball in $\mathbb{R}^{k}$ of radius $r$, centered at $P_{0}$. One of the principal ingredients in the proof of Theorem 1.1 is the following stability inequality.

Proposition 2.1. There exists a constant $C_{N}$ depending only on $N$ such that if $u \in W^{1, p}(M, N)$ is a p-harmonic map satisfying $u(M) \subset B\left(P_{0}, r\right)$, for some $P_{0} \in N$ and $0<r<C_{N}$, then we have, for any $\varphi \in W_{0}^{1, p}\left(M, \mathbb{R}^{k}\right)$,

$$
\int_{M}|\nabla u|^{p}|\varphi|^{2} d g \leq 16 r^{2} \int_{M}|\nabla u|^{p-2}|\nabla \varphi|^{2} d g
$$

Proof. By a limiting argument, it suffices to prove the proposition for $\varphi \in C_{0}^{\infty}\left(M, \mathbb{R}^{k}\right)$ since this space is dense in $W_{0}^{1, p}\left(M, \mathbb{R}^{k}\right)$. Let then $\varphi \in C_{0}^{\infty}\left(M, \mathbb{R}^{k}\right)$ and take $|\varphi|^{2}\left(u-P_{0}\right)$ as testing function in the system $(1.2)$, we get

$$
\begin{gathered}
\int_{M}|\nabla u|^{p}|\varphi|^{2} d g \leq 2 \int_{M}|\nabla u|^{p-1}\left|u-P_{0}\right||\nabla \varphi||\varphi| d g \\
\quad+\int_{M}|\nabla u|^{p-2}|A(u)(\nabla u, \nabla u)|\left|u-P_{0}\right||\varphi|^{2} d g .
\end{gathered}
$$

Since $N$ is compact and $\left|u-P_{0}\right|<r$, we have by the bilinearity of the second fundamental form, $|A(u)(\nabla u, \nabla u)|\left|u-P_{0}\right| \leq C_{0} r|\nabla u|^{2}$, where $C_{0}$ is a constant depending on $N$. Then the last term in the right hand side of (2.1) satisfies

$$
\int_{M}|\nabla u|^{p-2}|A(u)(\nabla u, \nabla u)|\left|u-P_{0}\right||\varphi|^{2} d g \leq C_{0} r \int_{M}|\nabla u|^{p}|\varphi|^{2} d g .
$$

On the other hand, by the Cauchy-Schwarz inequality we have

$$
\begin{gathered}
2 \int_{M}|\nabla u|^{p-1}\left|u-P_{0}\right||\nabla \varphi||\varphi| d g \leq \\
2 r\left(\int_{M}|\nabla u|^{p}|\varphi|^{2} d g\right)^{\frac{1}{2}}\left(\int_{M}|\nabla u|^{p-2}|\nabla \varphi|^{2} d g\right)^{\frac{1}{2}} .
\end{gathered}
$$

It follows from (2.1), (2.2) and (2.3) that

$$
\left(1-C_{0} r\right) \int_{M}|\nabla u|^{p}|\varphi|^{2} d g \leq 2 r\left(\int_{M}|\nabla u|^{p}|\varphi|^{2} d g\right)^{\frac{1}{2}}\left(\int_{M}|\nabla u|^{p-2}|\nabla \varphi|^{2} d g\right)^{\frac{1}{2}},
$$


which gives, if we suppose $C_{0} r<\frac{1}{2}$,

$$
\left(\int_{M}|\nabla u|^{p}|\varphi|^{2} d g\right)^{\frac{1}{2}} \leq 4 r\left(\int_{M}|\nabla u|^{p-2}|\nabla \varphi|^{2} d g\right)^{\frac{1}{2}} .
$$

The proposition is then proved.

For the proof Theorem 1.1 we need also two lemmas. The first one concerns some inequalities on Euclidean spaces that we will prove for the convenience of the reader.

Lemma 2.1. Let $V$ be a real vector space endowed with an inner product. For $X, Y \in V$, we denote by $X \cdot Y$ the inner product of $X$ and $Y$, and by $|X|=\sqrt{X \cdot X}$ the associated norm of $X$. Then for any $q \geq 0$, and for any $X, Y \in V$, we have

$$
\left(|X|^{q} X-|Y|^{q} Y\right) \cdot(X-Y) \geq \frac{1}{2}\left(|X|^{q}+|Y|^{q}\right)|X-Y|^{2}
$$

and

$$
\left.|| X\right|^{q} X-|Y|^{q} Y\left|\leq(q+1)\left(|X|^{q}+|Y|^{q}\right)\right| X-Y \mid
$$

Proof. Let us first prove (2.4). We may suppose, without loss of generality, that $|X|=1$. Let $V_{X, Y}$ be a 2-dimensional vector subspace of $V$ containing the vectors $X$ and $Y$. Set $e_{1}=X$ and choose a vector $e_{2} \in V_{X, Y}$ such that $\left\{e_{1}, e_{2}\right\}$ is an orthonormal basis of $V_{X, Y}$. Taking coordinates with respect to this basis, we have

$$
X=(1,0) \text { and } Y=(r \cos \theta, r \sin \theta) \text {, with } r=|Y|, \theta \in[0,2 \pi] .
$$

Then inequality (2.4) can be written

$$
1+r^{q+2}-r\left(1+r^{q}\right) \cos \theta \geq \frac{1}{2}\left(1+r^{q}\right)\left(1+r^{2}-2 r \cos \theta\right),
$$

which is equivalent to

$$
1+r^{q+2}-r^{q}-r^{2} \geq 0 .
$$

But the last inequality is always true since $1+r^{q+2}-r^{q}-r^{2}=\left(1-r^{2}\right)\left(1-r^{q}\right)$ and $q \geq 0$. This proves $(2.4)$.

Now, to prove (2.5) we set $F(Z)=|Z|^{q} Z$. Then by the mean value theorem, we have

$$
\left\|\left.X\right|^{q} X-|Y|^{q} Y|=| F(X)-F(Y)\left|\leq \sup _{0 \leq t \leq 1}\|d F(t X+(1-t) Y)\|\right| X-Y \mid,\right.
$$

where $d F(Z): V \rightarrow V$ is the differential of $F$ at $Z$, and where we denote by $\|L\|$ the norm of any bounded linear map $L: V \rightarrow V$. A straightforward computation gives, for any $Z \in V$,

$$
\|d F(Z)\| \leq(q+1)|Z|^{q},
$$


which implies

This proves $(2.5)$.

$$
\begin{gathered}
\left.|| X\right|^{q} X-|Y|^{q} Y\left|\leq(q+1) \sup _{0 \leq t \leq 1}\right| t X+\left.(1-t) Y\right|^{q}|X-Y| \\
\leq(q+1) \sup _{0 \leq t \leq 1}(t|X|+(1-t)|Y|)^{q}|X-Y| \\
\leq(q+1) \max \left(|X|^{q},|Y|^{q}\right)|X-Y| \\
\leq(q+1)\left(|X|^{q}+|Y|^{q}\right)|X-Y|
\end{gathered}
$$

In the following lemma, we prove an inequality satisfied by the second fundamental form of $N$. For any $y \in N$, we denote by $T_{y} N \subset \mathbb{R}^{k}$ the tangent space of $N$ at $y$, and $A(y): T_{y} N \times T_{y} N \rightarrow T_{y} N^{\perp}$ the second fondamental form of $N$ at $y$.

Lemma 2.2. There is a constant $C$ depending only on $N$ such that, for any $y, z \in N$, and for any $Y \in T_{y} N, Z \in T_{z} N$, we have

$$
\begin{gathered}
|A(y)(Y, Y)-A(z)(Z, Z)| \leq \\
C\left(|Y|^{2}+|Z|^{2}\right)|y-z|+C(|Y|+|Z|)|Y-Z|,
\end{gathered}
$$

where $|$.$| denotes the Euclidean norm in \mathbb{R}^{k}$.

Proof. Since $N$ is smooth and compact, there exist $\delta>0$, and a finite number of points $y_{1}, \cdots, y_{K} \in N$ such that

$$
N \subset \bigcup_{\nu=1}^{K} B\left(y_{\nu}, \frac{\delta}{2}\right),
$$

and for each $\nu=1, \cdots, K$, a smooth orthonormal frame $\left\{e_{i}(y)\right\}_{1 \leq i \leq n}$ on $N \cap$ $B\left(y_{\nu}, \delta\right)$ of the tangent space of $N$ at $y \in N \cap B\left(y_{\nu}, \delta\right)$. Let $y, z \in N$ and $Y \in$ $T_{y} N, Z \in T_{z} N$. If $|y-z| \geq \frac{\delta}{2}$, then we have by the bilinearity of the second fondamental form :

$$
\begin{gathered}
|A(y)(Y, Y)-A(z)(Z, Z)| \leq C\left(|Y|^{2}+|Z|^{2}\right) \\
\leq 2 C \delta^{-1}\left(|Y|^{2}+|Z|^{2}\right)|y-z|
\end{gathered}
$$

where $C$ is a positive constant depending only on $N$. This proves $(2.6)$ in this case. Now suppose that $|y-z|<\frac{\delta}{2}$. Then $y, z \in B\left(y_{\nu_{0}}, \delta\right)$ for some $1 \leq \nu_{0} \leq K$, and consider a smooth orthonormal frame $\left\{e_{i}\right\}_{1 \leq i \leq n}$ on $N \cap B\left(y_{\nu_{0}}, \delta\right)$. For each $i, j=1, \cdots, n$, consider the map $F_{i j}: B\left(y_{\nu_{0}}, \delta\right) \cap N \rightarrow \mathbb{R}^{k}$ defined by

$$
F_{i j}(x)=A(x)\left(e_{i}(x), e_{j}(x)\right), x \in B\left(y_{\nu_{0}}, \delta\right) \cap N .
$$

Since $F_{i j}$ is a smooth map, then we have by the mean value theorem

$$
\left|F_{i j}(y)-F_{i j}(z)\right| \leq C|y-z|
$$

for a constant $C$ depending only on $N$, that's

$$
\left|A(y)\left(e_{i}(y), e_{j}(y)\right)-A(z)\left(e_{i}(z), e_{j}(z)\right)\right| \leq C|y-z| .
$$


We have also by smoothness of the map $F_{i j},\left|F_{i j}(y)\right| \leq C,\left|F_{i j}(z)\right| \leq C$, that's

$$
\left|A(y)\left(e_{i}(y), e_{j}(y)\right)\right| \leq C \text { and }\left|A(z)\left(e_{i}(z), e_{j}(z)\right)\right| \leq C .
$$

If we take two tangent vectors $Y \in T_{y} N$ and $Z \in T_{z} N$, then $Y=\sum_{i=1}^{n} \alpha_{i} e_{i}(y)$ and $Z=\sum_{i=1}^{n} \beta_{i} e_{i}(z)$ for some $\left(\alpha_{1}, \cdots, \alpha_{n}\right) \in \mathbb{R}^{n}$ and $\left(\beta_{1}, \cdots, \beta_{n}\right) \in \mathbb{R}^{n}$. Set $\alpha=\left(\alpha_{1}, \cdots, \alpha_{n}\right)$ and $\beta=\left(\beta_{1}, \cdots, \beta_{n}\right)$, then

$$
|Y|=|\alpha|,|Z|=|\beta| \text {. }
$$

Now, we have

$$
A(y)(Y, Y)-A(z)(Z, Z)=\sum_{i, j=1}^{n}\left(\alpha_{i} \alpha_{j} A(y)\left(e_{i}(y), e_{j}(y)\right)-\beta_{i} \beta_{j} A(z)\left(e_{i}(z), e_{j}(z)\right)\right) .
$$

Then

$$
\begin{gathered}
|A(y)(Y, Y)-A(z)(Z, Z)| \leq \sum_{i, j=1}^{n}\left|\alpha_{i} \alpha_{j}\right|\left|A(y)\left(e_{i}(y), e_{j}(y)\right)-A(z)\left(e_{i}(z), e_{j}(z)\right)\right| \\
+\sum_{i, j=1}^{n}\left|\alpha_{i} \alpha_{j}-\beta_{i} \beta_{j}\right|\left|A(z)\left(e_{i}(z), e_{j}(z)\right)\right|
\end{gathered}
$$

which gives by using (2.7) and (2.8)

$$
\begin{gathered}
|A(y)(Y, Y)-A(z)(Z, Z)| \leq C \sum_{i, j=1}^{n}\left|\alpha_{i} \alpha_{j}\right||y-z|+C \sum_{i, j=1}^{n}\left|\alpha_{i} \alpha_{j}-\beta_{i} \beta_{j}\right| \\
\leq C \sum_{i, j=1}^{n}\left|\alpha_{i} \alpha_{j}\right||y-z|+C \sum_{i, j=1}^{n}\left|\alpha_{i}\right|\left|\alpha_{j}-\beta_{j}\right|+C \sum_{i, j=1}^{n}\left|\beta_{j}\right|\left|\alpha_{i}-\beta_{i}\right| \\
\leq C|\alpha|^{2}|y-z|+C(|\alpha|+|\beta|)|\alpha-\beta| .
\end{gathered}
$$

On the other hand, we have

$$
\begin{gathered}
|Y-Z|=\left|\sum_{i=1}^{n} \alpha_{i} e_{i}(y)-\beta_{i} e_{i}(z)\right| \geq\left|\sum_{i=1}^{n}\left(\alpha_{i}-\beta_{i}\right) e_{i}(y)\right|-\left|\sum_{i=1}^{n} \beta_{i}\left(e_{i}(y)-e_{i}(z)\right)\right| \\
\geq|\alpha-\beta|-\sum_{i=1}^{n}\left|\beta_{i}\right|\left|e_{i}(y)-e_{i}(z)\right| .
\end{gathered}
$$

But by using again the mean value theorem, we have, for any $i=1, \cdots, n$,

$$
\left|e_{i}(y)-e_{i}(z)\right| \leq C|y-z|
$$


where $C$ depends only on $N$. Hence it follows from (2.11) that

$$
|Y-Z| \geq|\alpha-\beta|-C|\beta||y-z|
$$

that's

$$
|\alpha-\beta| \leq|Y-Z|+C|\beta||y-z| .
$$

Combining this inequality with $(2.10)$ gives

$$
|A(y)(Y, Y)-A(z)(Z, Z)| \leq C\left(|\alpha|^{2}+|\beta|^{2}\right)|y-z|+C(|\alpha|+|\beta|)|Y-Z|,
$$

and since by $(2.9)$ we have $|\alpha|=|Y|,|\beta|=|Z|$, we finally obtain

$$
|A(y)(Y, Y)-A(z)(Z, Z)| \leq C\left(|Y|^{2}+|Z|^{2}\right)|y-z|+C(|Y|+|Z|)|Y-Z| .
$$

This proves the lemma.

We are now in position to prove Theorem 1.1 .

Proof of Theorem 1.1. Let $u, v \in W^{1, p}(M, N)$ be $p$-harmonic maps such that $u=$ $v$ on $\partial M$. Since $u$ and $v$ are solutions of system (1.2), then we have for any $\varphi \in W_{0}^{1, p}\left(M, \mathbb{R}^{k}\right) \cap L^{\infty}\left(M, \mathbb{R}^{k}\right)$,

$$
\left.\int_{M}|\nabla u|^{p-2} \nabla u \cdot \nabla \varphi d g=\int_{M}|\nabla u|^{p-2} A(u)(\nabla u, \nabla u)\right) \cdot \varphi d g
$$

and

$$
\left.\int_{M}|\nabla v|^{p-2} \nabla v \cdot \nabla \varphi d g=\int_{M}|\nabla v|^{p-2} A(v)(\nabla v, \nabla v)\right) \cdot \varphi d g .
$$

Taking the difference of the last two equations, and choosing $\varphi=u-v \in$ $W_{0}^{1, p}\left(M, \mathbb{R}^{k}\right) \cap L^{\infty}\left(M, \mathbb{R}^{k}\right)$ (since $u-v=0$ on $\left.\partial M\right)$, we get

$$
\begin{gathered}
\int_{M}\left(|\nabla u|^{p-2} \nabla u-|\nabla v|^{p-2} \nabla v\right) \cdot \nabla(u-v) d g= \\
\int_{M}\left(|\nabla u|^{p-2} A(u)(\nabla u, \nabla u)-|\nabla v|^{p-2} A(v)(\nabla v, \nabla v)\right) \cdot(u-v) d g .
\end{gathered}
$$

By choosing a local orthonormal frame on $M$, we can identify $\nabla u$ and $\nabla v$ with vectors in $\mathbb{R}^{\nu}$ (endowed with its usual inner product), where $\nu=m n$. Then inequality (2.4) of Lemma 2.1 applied to $X=\nabla u$ and $Y=\nabla v$, with $V=\mathbb{R}^{\nu}$ and $q=p-2$, gives

$$
\left(|\nabla u|^{p-2} \nabla u-|\nabla v|^{p-2} \nabla v\right) \cdot \nabla(u-v) \geq \frac{1}{2}\left(|\nabla u|^{p-2}+|\nabla v|^{p-2}\right)|\nabla(u-v)|^{2} .
$$


Then it follows from $(2.12)$ that

$$
\begin{gathered}
\int_{M}\left(|\nabla u|^{p-2}+|\nabla v|^{p-2}\right)|\nabla(u-v)|^{2} d g \leq \\
\left.2 \int_{M}|| \nabla u\right|^{p-2} A(u)(\nabla u, \nabla u)-|\nabla v|^{p-2} A(v)(\nabla v, \nabla v)|| u-v \mid d g .
\end{gathered}
$$

On the other hand, taking again a local orthonormal frame $\left\{e_{j}\right\}_{1 \leq j \leq m}$ on $M$, we recall that

$$
A(u)(\nabla u, \nabla u)=\sum_{j=1}^{m} A(u)\left(\nabla_{e_{j}} u, \nabla_{e_{j}} u\right)
$$

and

$$
A(v)(\nabla v, \nabla v)=\sum_{j=1}^{m} A(v)\left(\nabla_{e_{j}} v, \nabla_{e_{j}} v\right) .
$$

Then applying Lemma 2.2 with $y=u, z=v$, and $Y=|\nabla u|^{\frac{p-2}{2}} \nabla_{e_{i}} u, Z=$ $|\nabla v|^{\frac{p-2}{2}} \nabla_{e_{i}} v$, we have

$$
\begin{gathered}
\left.|| \nabla u\right|^{p-2} A(u)(\nabla u, \nabla u)-|\nabla v|^{p-2} A(v)(\nabla v, \nabla v) \mid \leq \\
\left.\sum_{i=1}^{m}|| \nabla u\right|^{p-2} A(u)\left(\nabla_{e_{i}} u, \nabla_{e_{i}} u\right)-|\nabla v|^{p-2} A(v)\left(\nabla_{e_{i}} v, \nabla_{e_{i}} v\right) \mid \leq \\
C \sum_{i=1}^{m}\left(|\nabla u|^{p-2}\left|\nabla_{e_{i}} u\right|^{2}+|\nabla v|^{p-2}\left|\nabla_{e_{i}} v\right|^{2}\right)|u-v| \\
+\left.C \sum_{i=1}^{m}\left(|\nabla u|^{\frac{p-2}{2}}\left|\nabla_{e_{i}} u\right|+|\nabla v|^{\frac{p-2}{2}}\left|\nabla_{e_{i}} v\right|\right)|| \nabla u\right|^{\frac{p-2}{2}} \nabla_{e_{i}} u-|\nabla v|^{\frac{p-2}{2}} \nabla_{e_{i}} v \mid .
\end{gathered}
$$

But we have

$$
\sum_{i=1}^{m}\left(|\nabla u|^{p-2}\left|\nabla_{e_{i}} u\right|^{2}+|\nabla v|^{p-2}\left|\nabla_{e_{i}} v\right|^{2}\right)|u-v|=\left(|\nabla u|^{p}+|\nabla v|^{p}\right)|u-v|
$$

and by Cauchy-Schwarz inequality we have

$$
\begin{aligned}
& \left.\sum_{i=1}^{m}\left(|\nabla u|^{\frac{p-2}{2}}\left|\nabla_{e_{i}} u\right|+|\nabla v|^{\frac{p-2}{2}}\left|\nabla_{e_{i}} v\right|\right)|| \nabla u\right|^{\frac{p-2}{2}} \nabla_{e_{i}} u-|\nabla v|^{\frac{p-2}{2}} \nabla_{e_{i}} v \mid \leq \\
& \left(\sum_{i=1}^{m}|\nabla u|^{p-2}\left|\nabla_{e_{i}} u\right|^{2}\right)^{1 / 2} \times\left(\left.\sum_{i=1}^{m}|| \nabla u\right|^{\frac{p-2}{2}} \nabla_{e_{i}} u-\left.|\nabla v|^{\frac{p-2}{2}} \nabla_{e_{i}} v\right|^{2}\right)^{1 / 2} \\
& +\left(\sum_{i=1}^{m}|\nabla v|^{p-2}\left|\nabla_{e_{i}} v\right|^{2}\right)^{1 / 2} \times\left(\left.\sum_{i=1}^{m}|| \nabla u\right|^{\frac{p-2}{2}} \nabla_{e_{i}} u-\left.|\nabla v|^{\frac{p-2}{2}} \nabla_{e_{i}} v\right|^{2}\right)^{1 / 2}
\end{aligned}
$$




$$
=\left.\left(|\nabla u|^{\frac{p}{2}}+|\nabla v|^{\frac{p}{2}}\right)|| \nabla u\right|^{\frac{p-2}{2}} \nabla u-|\nabla v|^{\frac{p-2}{2}} \nabla v \mid .
$$

Hence it follows from $(2.14)$ that

$$
\begin{gathered}
\left.|| \nabla u\right|^{p-2} A(u)(\nabla u, \nabla u)-|\nabla v|^{p-2} A(v)(\nabla v, \nabla v) \mid \leq \\
C\left(|\nabla u|^{p}+|\nabla v|^{p}\right)|u-v|+\left.C\left(|\nabla u|^{\frac{p}{2}}+|\nabla v|^{\frac{p}{2}}\right)|| \nabla u\right|^{\frac{p-2}{2}} \nabla u-|\nabla v|^{\frac{p-2}{2}} \nabla v \mid .
\end{gathered}
$$

By identifying the vectors $\nabla u$ and $\nabla v$ with vectors in $\mathbb{R}^{m n}$, and applying inequality (2.5) in Lemma 2.1 with $V=\mathbb{R}^{m n}, X=\nabla u, Y=\nabla v$, and $q=\frac{p-2}{2}$, we have

$$
\left.|| \nabla u\right|^{\frac{p-2}{2}} \nabla u-|\nabla v|^{\frac{p-2}{2}} \nabla v\left|\leq \frac{p}{2}\left(|\nabla u|^{\frac{p-2}{2}}+|\nabla v|^{\frac{p-2}{2}}\right)\right| \nabla u-\nabla v \mid .
$$

Combining this inequality with (2.15), we get

$$
\begin{gathered}
\left.|| \nabla u\right|^{p-2} A(u)(\nabla u, \nabla u)-|\nabla v|^{p-2} A(v)(\nabla v, \nabla v) \mid \leq \\
C_{p}\left(|\nabla u|^{p}+|\nabla v|^{p}\right)|u-v|+C_{p}\left(|\nabla u|^{p-1}+|\nabla v|^{p-1}\right)|\nabla(u-v)|,
\end{gathered}
$$

where $C_{p}$ is a positive constant depending only on $N$ and $p$.

Putting (2.16) in (2.13), we obtain

$$
\begin{aligned}
\int_{M}\left(|\nabla u|^{p-2}\right. & \left.+|\nabla v|^{p-2}\right)|\nabla(u-v)|^{2} d g \leq C_{p} \int_{M}\left(|\nabla u|^{p}+|\nabla v|^{p}\right)|u-v|^{2} d g, \\
& +C_{p} \int_{M}\left(|\nabla u|^{p-1}+|\nabla v|^{p-1}\right)|\nabla(u-v)||u-v| d g
\end{aligned}
$$

which by the Cauchy-Schwarz inequality gives

$$
\begin{gathered}
\int_{M}\left(|\nabla u|^{p-2}+|\nabla v|^{p-2}\right)|\nabla(u-v)|^{2} d g \leq C_{p} \int_{M}\left(|\nabla u|^{p}+|\nabla v|^{p}\right)|u-v|^{2} d g \\
+C_{p}\left(\int_{M}\left(|\nabla u|^{p-2}+|\nabla v|^{p-2}\right)|\nabla(u-v)|^{2} d g\right)^{1 / 2} \\
\times\left(\int_{M}\left(|\nabla u|^{p}+|\nabla v|^{p}\right)|u-v|^{2} d g\right)^{1 / 2}
\end{gathered}
$$

Now, if the constant $\varepsilon_{0}$ in Theorem 1.1 is sufficiently small, then the geodesic ball $B\left(P_{0}, \varepsilon_{1}\right)$ in $N$ is included in the Euclidean ball $B\left(P_{0}, r\right)$ in $\mathbb{R}^{k}$ with $r$ as in Proposition 2.1. Applying Proposition 2.1 to both $u$ and $v$ with $\varphi=u-v \in$ $W_{0}^{1, p}\left(M, \mathbb{R}^{k}\right)$, and taking the sum, we have

$$
\int_{M}\left(|\nabla u|^{p}+|\nabla v|^{p}\right)|u-v|^{2} d g \leq
$$




$$
16 r^{2} \int_{M}\left(|\nabla u|^{p-2}+|\nabla v|^{p-2}\right)|\nabla(u-v)|^{2} d g .
$$

Combining this inequality with (2.17), we finally obtain

$$
\begin{gathered}
\int_{M}\left(|\nabla u|^{p-2}+|\nabla v|^{p-2}\right)|\nabla(u-v)|^{2} d g \leq \\
C_{p}\left(16 r^{2}+4 r\right) \int_{M}\left(|\nabla u|^{p-2}+|\nabla v|^{p-2}\right)|\nabla(u-v)|^{2} d g .
\end{gathered}
$$

Thus if $r$ is small enough, we obtain $\nabla u-\nabla v=0$, and then $u=v$ since $u=v$ on $\partial M$. This proves Theorem 1.1 .

\section{ENERGY MINIMIZING MAPS WITH SMALL RANGE}

Our goal in this section is the proof of Theorem 1.2. To this end, we need the following proposition on the existence of a minimizing $p$-harmonic map, agreeing with $u$ on the boundary of the domain, and having its image in the same geodesic ball as $u$. In what follows, we set

$$
r_{N}=\inf \left(i_{N}, \frac{\pi}{2 \kappa}\right)
$$

where $i_{N}$ is the injectivity radius of the manifold $N$ and $\kappa \geq 0$ is an upper bound of the sectional curvature of $N$.

Proposition 3.1. Let $0<r<r_{N}$, and let $u \in W^{1, p}(D, N)$ satisfying $u(D) \subset$ $B\left(P_{0}, r\right)$, where $D \subset M$ is an open set with smooth boundary $\partial D$, and $B\left(P_{0}, r\right)$ is a geodesic ball in $N$ of radius $r$, centered at some point $P_{0} \in N$. Then there exists a p-harmonic map $v \in W^{1, p}(D, N)$ such that $v=u$ on $\partial D, v(D) \subset B\left(P_{0}, r\right)$ and satisfying

$$
\int_{D}|\nabla v|^{p} d g \leq \int_{D}|\nabla w|^{p} d g
$$

for all $w \in W^{1, p}(D, N)$ with $w(D) \subset B\left(P_{0}, r\right)$ and $w=u$ on $\partial D$.

Proof. The proof is an adaptation of a similar result by S. Hildebrandt et al [7] in the harmonic case $(p=2)$. The existence of $v$ relies on classical variational arguments, so the main difficulty is to prove that $v$ is $p$-harmonic. Fix $r_{1}$ such that $r<r_{1}<r_{N}$. Since the functional $E_{p}$ is bounded from below, there is a minimizing sequence $\left(v_{l}\right)$ of the $p$-energy in $W^{1, p}(D, N)$ such that $v_{l}(D) \subset \overline{B\left(P_{0}, r_{1}\right)}$ and $v_{l}=u$ on $\partial D$. Up to a subsequence, we may suppose that $\left(v_{l}\right)$ converges weakly in $W^{1, p}\left(D, \mathbb{R}^{k}\right)$ and strongly in $L^{p}\left(D, \mathbb{R}^{k}\right)$ to some $v \in W^{1, p}(D, N)$, with $v(D) \subset$ 
$\overline{B\left(P_{0}, r_{1}\right)}$ and $v=u$ on $\partial D$. Moreover, by lower semi-continuity of the $p$-energy functional, we have

$$
\int_{D}|\nabla v|^{p} d g \leq \liminf _{l \rightarrow \infty} \int_{D}\left|\nabla v_{l}\right|^{p} d g
$$

This proves that $v$ is minimizing the $p$-energy on $D$ among maps having their values in $\overline{B\left(P_{0}, r_{1}\right)}$ and agreeing with $u$ on $\partial D$. It remains to show that $v(D) \subset B\left(P_{0}, r\right)$ and that $v$ is $p$-harmonic in $D$. We follow the arguments of [7], and we shall prove that $v$ satisfies the system (1.1) (in the weak sense ) in normal coordinates around $P_{0}$. Let $v=\left(v^{1}, \cdots, v^{n}\right)$ be the expression of $v$ in such normal coordinates. Then we have to prove that, for any $\varphi=\left(\varphi^{1}, \cdots, \varphi^{n}\right) \in W_{0}^{1, p}\left(D, \mathbb{R}^{n}\right) \cap L^{\infty}\left(D, \mathbb{R}^{n}\right)$,

$$
\int_{D}|d v|^{p-2} \nabla v^{i} \nabla \varphi^{i} d g=\int_{D}|d v|^{p-2} \Gamma_{k l}^{j}(v) \varphi^{j} \nabla v^{k} \nabla v^{l} d g
$$

First let us prove that $v(D) \subset B\left(P_{0}, r\right)$, that's, $|v|<r$ (recall that we are working in normal coordinates around $\left.P_{0}\right)$. Let $0 \leq \eta \in W_{0}^{1, p}(D, \mathbb{R}) \cap L^{\infty}(D, \mathbb{R})$ and consider the function $v_{t}=\exp _{P_{0}}((1-t \eta) v)$, with $0 \leq t \leq\|\eta\|_{\infty}^{-1}$. Then $v_{t} \in W^{1, p}(D, N), v_{t}=u$ on $\partial D, v_{t}(D) \subset \overline{B\left(P_{0}, r_{1}\right)}$, and $v_{0}=v$. Hence, since $v$ is minimizing, we have $E_{p}(v) \leq E_{p}\left(v_{t}\right)$ for any $0 \leq t \leq\|\eta\|_{\infty}^{-1}$. If we take the derivative with respect to $t$ at $t=0$, we get

which gives (after some computations)

$$
\left.\frac{d}{d t} E_{p}\left(v_{t}\right)\right|_{t=0} \geq 0
$$

$$
\int_{D}|d v|^{p-2} \nabla v^{i} \nabla\left(\eta v^{i}\right) d g-\int_{D} \eta|d v|^{p-2} \Gamma_{k l}^{j}(v) v^{j} \nabla v^{k} \nabla v^{l} d g \leq 0 .
$$

Now if we choose $\eta=\max \left(|v|^{2}-r^{2}, 0\right)$, then it is easy to see that $\eta \in$ $W_{0}^{1, p}(D, \mathbb{R}) \cap L^{\infty}(D, \mathbb{R})$ and that $\nabla \eta=2 \nabla v \cdot v$ if $|v| \geq r$ and $\nabla \eta=0$ if $|v| \leq r$. Replacing $\eta$ in the last inequality, we obtain

$$
\frac{1}{2} \int_{D}|d v|^{p-2}|\nabla \eta|^{2} d g+\int_{D} \eta|d v|^{p-2}\left(|\nabla v|^{2}-\Gamma_{k l}^{j}(v) v^{j} \nabla v^{k} \nabla v^{l}\right) d g \leq 0 .
$$

Since $|v| \leq r_{1}$ and $r_{1}<r_{N}$, we have, according to a result proved in [8] (inequality (6.11) ),

$$
|\nabla v|^{2}-\Gamma_{k l}^{j}(v) v^{j} \nabla v^{k} \nabla v^{l} \geq 0 .
$$

Thus we obtain

$$
\int_{D}|d v|^{p-2}|\nabla \eta|^{2} d g \leq 0
$$

which gives $\nabla \eta=0$, that's, $|v|<r$. It remains then to prove (3.2). Let $\varphi \in$ $W_{0}^{1, p}\left(D, \mathbb{R}^{n}\right) \cap L^{\infty}\left(D, \mathbb{R}^{n}\right)$, and consider the map $v_{t}=\exp _{P_{0}}(v+t \varphi)$. Then since $|v|<r<r_{1}$, we have $v_{t}(D) \subset B\left(P_{0}, r_{1}\right)$, for any $t \in(-\delta, \delta)$, with $\delta=$ $\left(r_{1}-r\right)\|\varphi\|_{\infty}^{-1}>0$, and $v_{t}=u$ on $\partial D$. Since $v$ is minimizing the $p$-energy functional among $W^{1, p}$ maps having their values in the ball $\overline{B\left(P_{0}, r_{1}\right)}$ and agreeing with $u$ on 
$\partial D$, we have $E_{p}(v) \leq E_{p}\left(v_{t}\right)$ for any $t \in(-\delta, \delta)$. By taking the derivative with respect to $t$ of $E_{p}\left(v_{t}\right)$ at $t=0$, we obtain

$$
\left.\frac{d}{d t} E_{p}\left(v_{t}\right)\right|_{t=0}=0
$$

which gives (3.2), This achieves the proof of Proposition 3.1,

Proof of Theorem 1.2. Let $\varepsilon_{0}$ as in Theorem 1.1 and let $\varepsilon_{1}>0$ such that

$$
\varepsilon_{1}<\inf \left(i_{N}, \frac{\pi}{4 \kappa}, \varepsilon_{0}\right)
$$

where $i_{N}$ is the injectivity radius of the manifold $N$ and $\kappa \geq 0$ is an upper bound of the sectional curvature of $N$. Let $u$ as in Theorem 1.2 such that $u(\Omega) \subset B\left(P_{0}, \varepsilon_{1}\right)$, and let $D \subset \Omega$ a domain with smooth boundary $\partial D$. By Proposition 3.1, with $r=\varepsilon_{1}$, there is a $p$-harmonic map $v \in W^{1, p}(D, N)$ such that $v=u$ on $\partial D$, $v(D) \subset B\left(P_{0}, \varepsilon_{1}\right)$ and satisfying

$$
\int_{D}|\nabla v|^{p} d g \leq \int_{D}|\nabla w|^{p} d g
$$

for any $w \in W^{1, p}(D, N)$ with $w(D) \subset B\left(P_{0}, \varepsilon_{1}\right)$ and $w=u$ on $\partial D$. Since $v$ is minimizing the $p$-energy, then it is easy to see that $v$ is a stationary $p$-harmonic map. It follows from the regularity result of M. Fuchs [3] for stationary $p$-harmonic maps with image in geodesic balls of radius $r<\inf \left(i_{N}, \frac{\pi}{4 \kappa}\right)$, that $v$ is in $C^{1, \alpha}(D, N)$ for some $0<\alpha<1$. But by Theorem 1.1 we have $u=v$ since we are supposing $\varepsilon_{1}<\inf \left(i_{N}, \frac{\pi}{4 \kappa}, \varepsilon_{0}\right) \leq \varepsilon_{0}$. This achieves the proof of Theorem 1.2 .

\section{REFERENCES}

[1] F. Bethuel, On the singular set of stationary harmonic maps, Manuscripta Math. 78 (1993), 417-443.

[2] A. Fardoun, On weakly p-harmonic maps to a closed hemisphere, Manuscripta Math. 116 (2005), 57-69.

[3] M. Fuchs, Some resularity theorems for mappings which are stationary points for the p-energy functional, Analysis 9 (1989), 127-143.

[4] A. Gastel, J. Grotowski and M. Kronz, Removable singularities for p-harmonic maps : the subquadratic case, Adv. Geom. 5 (2005), 469-483.

[5] R. Hardt and F-H. Lin, Mapping minimizing the $L^{p}$ norm of the gradient, Comm. Pure Appl. Math. 11, (1987), 555-588.

[6] F. Hélein, Régularité des applications faiblement harmoniques entre une surface et une variété riemannienne, C. R. Acad. Sci. Paris Sr. I Math. 312 (1991), 591-596.

[7] S. Hildebrandt, H. Kaul and O. Widman, An existence theory for harmonic mappings of Riemannian manifolds, Acta Math., 138 (1977), 1-16. 
[8] S. Hildebrandt, Harmonic mappings of Riemannian manifolds, in : Harmonic mappings and minimal immersions, E. Guisti, ed. , Springer Lectures Notes in Mathematics, 1161 (1984).

[9] W. Jäger and H. Kaul, Uniqueness and stability of harmonic maps and their Jacobi fields, Manuscripta Math. 28 (1979), 269-291.

[10] S. Luckhauss, Partial Hölder continuity for minima of certain energies among maps into a Riemannian manifold, Indiana Univ. Math. J 37 (1988), 349-367.

[11] T. Rivière and M. Struwe, Partial regularity for harmonic maps, and related problems, Comm. Pure and App. Math. 61 (2008), 451-463.

[12] R. Schoen and K. Uhlenbeck, A regularity theory for harmonic maps, J. Differential Geom. 17 (1982), 307-335.

[13] P. Strzelecki, Regularity of generalized sphere valued p-harmonic maps with small mean oscillations, Manuscripta Math. 112 (2003), 473-487.

Laboratoire de Mathématiques, UMR 6205 CNRS, Université de Bretagne OCcidentale, 6 Avenue le Gorgeu, 29238 Brest Cedex 3, France 